\section{Comment on: Evaluation of early biomarkers of renal dysfunction in diabetic patients}

\author{
To the Editor
}

I have read with a great interest the article of "Evaluation of early biomarkers of renal dysfunction in diabetic patients" by Al-Hazmi et al. ${ }^{1}$

Diabetic nephropathy is one of the microvascular complications of diabetes mellitus. It has been linked to increase mortality in patients with diabetes mellitus. ${ }^{2}$ The surge of mortality in these patients progresses with the advancement of diabetic nephropathy. ${ }^{3}$ The standard biomarker of diabetic nephropathy is urine albumin/creatinine ratio. So, finding novel biomarkers that might detect the kidney damage early is a crucial advancement in medicine that might lead to early treatment and prevention of the disease progression.

The main result of the study was the significant increase of the studied biomarkers (urinary neutrophil gelatinase-associated lipocalin [uNGAL], urinary N-acetyle-beta-D-glucosaminidase [uNAG], and serum Cystatin C) in patients with diabetes mellitus and severely albuminuria as compared to those with moderately increased albuminuria, normal albuminuria, and normal control. This finding was similarly found in previous studies. ${ }^{4,5}$ Additionally, the study came with a very interesting finding that the level of uNGAL was significantly higher in the group with diabetes mellitus and normal albuminuria as compared to normal control. The authors have indicated that such result might allude to early discovery of kidney injury in patients with diabetes. Such result is fascinating, and it might open the door for researches in the future regarding the early discovery and management of diabetic nephropathy.

There were several drawbacks on the study, first the sample size was small. Secondly, there was no matching point between the 4 groups. For example, all the groups had different age, duration of diabetes and different glycemic control which might contribute to differences in the results. Furthermore, the medications which might affect the albumin secretion were not included in the study like ACEi, ARB, or sGLT2i. Finally, the issue of having a significant difference between a new biomarker in patients with normal albuminuria might not indicate an early disease since there no histological study to confirm that patients were suffering diabetic nephropathy. I agree such result creates a hypothesis, but on the other hand, it might simply mean that the biomarker is not specific.

In conclusion, diabetic nephropathy is a nasty complication of diabetes mellitus with an increase morbidity and mortality that necessitate early discovery of novel markers. The uNGAL might a be good biomarker to detect early renal dysfunction. However, a larger, and a well-organized study is needed in the future to validate their use in clinical practice.

\section{Abdulghani H. Alsaeed Endocrinology \& Diabetes Department Prince Sultan Military Medical City Riyadh, Kingdom of Saudi Arabia}

\section{Reply from the Author}

No reply was received from the Author.

\section{References}

1. Al-Hazmi SF, Gad HG, Alamoudi AA, Eldakhakhny BM, Binmahfoozv SK, Alhozali AM. Evaluation of early biomarkers of renal dysfunction in diabetic patients. Saudi Med J 2020; 41: 690-697.

2. Afkarian M, Sachs MC, Kestenbaum B, Hirsch IB, Tuttle KI, Himmelfarb J, et al. Kidney disease and increased mortality risk in type 2 Diabetes. J Am Soc Nephrol 2013; 24: 302-308

3. Bentata Y, Haddiya I, Latrech H, Serraj K. Progression of diabetic nephropathy, risk of end-stage renal disease and mortality in patients with type-1 diabetes Saudi J Kidney Dis Transpl 2013; 24: 392-402.

4. Siddiqi Z, Karoli R, Kaul A, Fatima J, Varshney S, Beg MS. Evaluation of neutrophil gelatinase-associated lipocalin and cystatin C as early markers of diabetic nephropathy. Ann Afr Med 2017; 16: 101-106.

5. Chena B, Lib Y, Liua Y, Zanga C, Wua M, Xu Z. Diagnostic value of neutrophil gelatinase-associated lipocalin in diabetic nephropathy: a meta-analysis. Renal Failure 2019: 41: 489-496 